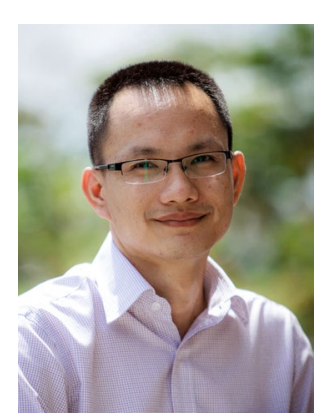

\title{
COVID-19 control in Vietnam
}

\author{
Early preparedness, contact tracing, isolation and testing, coupled with timely border closure, \\ physical distancing and community adherence, have been key measures in controlling \\ COVID-19 in Vietnam.
}

Credit: Le Van Tan

$\mathrm{V}$ ietnam is a lower-middle income country of 97 million people. Per capita, its GDP in 2018 was US $\$ 2,566$, and around US $\$ 150$ per person per year was spent on healthcare. The country shares a 1,300-km border with China, where SARS-CoV-2 first emerged. Yet, by the end of 2020, Vietnam had experienced only two peaks of community transmission (March-April and July-August) and reported a total of 1,465 PCR-confirmed cases of SARS-CoV-2 infection and 35 deaths. Here, I reflect on how Vietnam has succeeded in controlling the virus.

The experience Vietnam gained from previous epidemics, such as SARS-CoV-1 in 2003 and bird flu (H5N1) in 2004, was critical in informing its rapid response to COVID-19. On 16 January, well in advance of the first case of SARS-CoV-2 in the country, the Vietnamese Government issued the first diagnostic and management guidelines for COVID-19. The guidelines provided instructions on contact tracing and 14-day isolation of direct contacts (F1) of a confirmed case. This allowed Vietnam to successfully diagnose the first two epidemiologically linked COVID-19 cases (a father and son) on 23 January. The father arrived from Wuhan, China, to visit his son in Mekong Delta, representing the first instance of human-to-human transmission of SARS-CoV-2 outside of China. Their contacts were traced, isolated and tested; one was positive for SARS-CoV-2. Thus the first community transmission chain in the country was quickly identified and contained.

Immediately following the detection of these cases, Vietnam suspended all flights from Wuhan. At that time, the WHO advised against travel restrictions. Shortly after this, in February, the country also closed its border and banned international flights to and from China, extending this to the rest of the world in March.

Schools were closed early, initially for the Lunar New Year from late January to early February, but closures were extended to early May, when the first wave was successfully controlled. In parallel, physical distancing, wearing a face mask in public and in-country travel restrictions were applied with increasing stringency, with strong public support and adherence. Suspected transmission hotspots (for example, a city or a community) were locked down to stop community transmission. On 31 March, when community transmission was escalating and the total reported cases in Vietnam exceeded 200, the prime minister enacted a nationwide lockdown for 15 days, which was extended to 30 April for high-risk cities. Accordingly, non-essential businesses and public transport were shut down, and only essential travel between cities and provinces was allowed. Gatherings of $>2$ people in public places were prohibited. People were asked to not leave their houses unless it was for essential activities (for example, seeking medical care or buying food).

Nasal and throat swab testing by PCR was applied to all F1s (regardless of symptoms) and travelers entering Vietnam. Confirmed cases, their F1s (regardless of the PCR results) and travelers were all subjected to a minimum quarantine of 14 days at one of the government-run isolation centers, deployed across the country since March. Meanwhile, home isolation for a maximum of 14 days was mandatory for those in contact with an F1. By the end of 2020, 730,000 individuals had been quarantined at one of the isolation centers across Vietnam, and 1.7 million people had been tested for SARS-CoV-2. Mass testing coupled with a creative sample-pooling strategy, whereby nasal and throat swabs from 2-7 individuals were placed in one tube at collection, was key to the success of suppressing the second wave in central Vietnam between July and August (Thanh, T. T. et al. Preprint at medRxiv https:// doi.org/10.1101/2020.09.11.20192484, 2020).

An innovative communication strategy was developed to keep the public informed and safe. From the start of the pandemic, the government sent regular updates on COVID-19-preventive responses and the national situation via SMS texts. Mobile apps, especially those developed locally, such as
Bluezone, helped users to identify whether they were in contact with a confirmed case. In February, a popular song was given new lyrics, 'Ghen Co Vy' (Jealous Coronavirus), to raise public awareness of the disease and to promote good hygiene habits to reduce the risk of acquiring COVID-19. To date, the song has attracted $\sim 70$ million views.

The government has also sought to mitigate the socioeconomic impacts of COVID-19. From August, Vietnam slowly opened its borders to allow highly skilled workers and experts to enter the country, although everyone entering undergoes a compulsory 14-day isolation period in designated hotels or government facilities, with SARS-CoV-2 PCR screening on days 1 and 14. The government has arranged $>200$ flights to repatriate $>60,000$ citizens stuck abroad and issued financial assistance packages to support businesses and employees affected by COVID-19. Vietnam's GDP growth rate was $2.91 \%$ in 2020 - one of the highest rates in the world.

Collectively, early preparedness, contact tracing, isolation and mass PCR screening, coupled with timely border closure, physical distancing and community adherence, have been the key components that have determined the success of Vietnam's control of COVID-19. Heading into the second year of the pandemic, phase 1 clinical trials of locally developed vaccine candidates are ongoing in Vietnam, and 30 million doses of the Oxford-AstraZeneca vaccine have been ordered for 2021. Until then, the Government will continue to enforce the measures that gave it such exemplary control of COVID-19 in 2020.

\section{Le Van Tan (1D $凶$ \\ Oxford University Clinical Research Unit, Hospital for Tropical Diseases, Ho Chi Minh City, Vietnam.

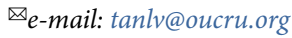

Published online: 24 February 2021 https://doi.org/10.1038/s41590-021-00882-9

Competing interests

The author declares no competing interests. 